\title{
Suanzaoren Formulae for Insomnia: Updated Clinical Evidence and Possible Mechanisms
}

\author{
Qi-Hui Zhou ${ }^{\dagger}$, Xiao-Li Zhou ${ }^{+}$, Meng-Bei Xu ${ }^{\dagger}$, Ting-Yu Jin, Pei-Qing Rong, \\ Guo-Qing Zheng* and Yan Lin*
}

Department of Neurology, The Second Affiliated Hospital and Yuying Children's Hospital of Wenzhou Medical University, Wenzhou, China

Insomnia disorder is a widespread and refractory disease. Semen Ziziphi Spinosae, Suanzaoren, a well-known Chinese herbal medicine, has been used for treating insomnia for thousands of years. Here, we aimed to assess the available evidence of Chinese herbal formulae that contains Suanzaoren (FSZR) for insomnia according to high-quality randomized controlled trials (RCTs) and reviewed their possible mechanisms based

OPEN ACCESS

Edited by: Luc Pieters,

University of Antwerp, Belgium

Reviewed by:

Anthony Booker,

University of Westminster,

United Kingdom

Germain Sotoing Taiwe,

University of Buea, Cameroon

*Correspondence:

Guo-Qing Zheng

gq_zheng@sohu.com

Yan Lin

linyanm@vip.sina.com

${ }^{\dagger}$ These authors have contributed equally to this work.

Specialty section:

This article was submitted to

Ethnopharmacology,

a section of the journal

Frontiers in Pharmacology

Received: 31 October 2017

Accepted: 23 January 2018

Published: 09 February 2018

Citation:

Zhou Q-H, Zhou X-L, XU M-B, Jin T-Y, Rong $P-Q$, Zheng $G-Q$ and Lin $Y$ (2018) Suanzaoren Formulae for Insomnia: Updated Clinical Evidence and Possible Mechanisms.

Front. Pharmacol. 9:76.

doi: 10.3389/fphar.2018.00076 on animal-based studies. Electronic searches were performed in eight databases from inception to November 2016. The primary outcome measures were polysomnography index and Pittsburgh sleep quality index. The secondary outcome measures were clinical effective rate and adverse events. The methodological quality of RCTs was assessed by Cochrane's collaboration tool, and only RCTs with positive for 4 out of 7 for the Cochrane risk of bias domains were included in analyses. Thirteen eligible studies with 1,454 patients were identified. Meta-analysis of high-quality RCTs showed that FSZR monotherapy was superior to placebo $(P<0.01)$; FSZR plus Diazepam was superior to Diazepam alone $(P<0.05)$; there were mixed results comparing FSZR with Diazepam $(P>0.05$ or $P<0.05)$. Furthermore, FSZR caused fewer side effects than that of Diazepam. Suanzaoren contains complex mixtures of phytochemicals including sanjoinine $A$, Jujuboside $A$, spinosin and other flavonoids, which has sedative and hypnotic functions primarily mediated by the GABAergic and serotonergic system. In conclusion, the findings of present study supported that FSZR could be an alternative treatment for insomnia in clinic. FSZR exerted sedative and hypnotic actions mainly through the GABAergic and serotonergic system.

Keywords: suanzaoren, Semen Ziziphi Spinosae, Chinese herbal medicine, insomnia, sedative and hypnotic actions

\section{INTRODUCTION}

Insomnia is characterized by sustained difficulties in initiating or maintaining sleep and cause significant impairment of daytime functioning (American Academy of Sleep Medicine, 2014). Based on the International Classification of Sleep Disorder (ICSD)-3 of the American Academy of Sleep Medicine, chronic insomnia disorder referred to these symptoms that cause clinically significant functional distress or impairment at least three nights per week for at least 3 months, excluding other medical or mental disorders (Morin et al., 2006b). Insomnia is the most common sleep complaint, about one third general population worldwide experiencing insomnia symptoms 
accompanied by daytime dysfunction consequences and $\sim 50 \%$ of patients having a chronic course (Morin et al., 2006b; Buysse, 2013). Persistent insomnia is associated with depression, anxiety disorders, suicide, drug/alcohol abuse, accidents, and cardiovascular disease (Baglioni et al., 2011; Fernandez-Mendoza and Vgontzas, 2013). Moreover, insomnia reduces the quality of life of the patients, and results in increasing healthcare cost and utilization (Leger and Bayon, 2010). At least 90\% of insomniarelated costs are resulted from the work absences and reduced productivity (Daley et al., 2009). The treatments of insomnia include pharmacological therapies, psychological and behavioral therapies, and complementary and alternative therapies (CAM) (Krystal, 2009). Benzodiazepines, non-benzodiazepine hypnotics and melatonin receptor agonists are the primary pharmacological therapy. Additionally, the melatonin receptor agonist ramelteon, orexin receptor antagonist suvorexant, and the antidepressant doxepin also have FDA approval for insomnia therapy (Asnis et al., 2015). However, the limited use of these pharmacological treatments is due to the undesirable side-effects such as performance and memory impairment, residual sedation, falls, undesired behaviors during sleep, somatic symptoms, and drug interactions (Wilt et al., 2016). Psychological and behavioral therapies for insomnia are well supported by empirical evidence (Morin et al., 2006a), but they have remained underutilized because of requirement of significant training and long-term implementation (Trauer et al., 2015). Thus, there are rising numbers of insomniac patients who seek to various kinds of CAM around the world.

Traditional Chinese medicine (TCM), as a main part of CAM, includes Chinese herbal medicine (CHM), acupuncture, meditation and massage (Sarris et al., 2011; Zhao, 2013; Liu et al., 2015). A survey in Hong Kong Chinese reported that the most commonly used CAM modalities was CHM (Yeung et al., 2014). CHM formulae (Fufang) are a combination of several CHMs according to TCM theory Jun-Chen-Zuo-Shi, known as emperor-miniser-assisstant-courier, first recorded by Huangdi Neijing (Inner Canon of the Yellow Emperor; Fan et al., 2006). $\mathrm{CHM}$ has been used for treating insomnia in China for thousands of years (Li and Deng, 1995), and is still used today, both in China and elsewhere around the world increasingly (Chen et al., 2009; Frass et al., 2012). Semen ziziphi spinosae, spine date seed, suanzaoren (SZR), the dried seed of Ziziphus jujuba Mill. var. spinosa (Bunge) Hu ex H. F. Chou (Huang et al., 2016; Rodríguez and Rodríguez, 2017), is one of the most popular CHMs and has a long history of use in Chinese medicine (Yan, 2010; Yeh et al., 2011; Ni et al., 2015; Rodríguez and Rodríguez, 2017; Shergis et al., 2017; Singh and Zhao, 2017). SZR was first recorded in the Shennong Bencao Jing (Shennong's Classic of Materia Medica), the earliest medicine monograph of China written 2500 years ago ( $\mathrm{Gu}, 2007)$. SZR is the most frequently used single herb for treating insomnia (Lei et al., 2015). Systematic reviews of CHM for insomnia have demonstrated that SZR is also the most frequently used herb in randomized controlled trials (RCTs) (Yeung et al., 2012; Ni et al., 2015). Spinosin and jujubosides are the main active compounds of SZR contributing their sedative and hypnotic effects on insomnia (Peng et al., 2000; Li et al., 2003; Jiang et al., 2007). SZR have been widely used in many standard Chinese formulae for insomnia (Li, 2006). In particular, SZR decoction is a well-known classic Chinese herbal formula, and has been used for treating insomnia for more than thousand years, first recorded in Jingui Yaolue (Synopsis of Prescriptions of the Golden Chamber) by Zhang Zhongjing (AD 152-219). Furthermore, modern pharmacological study indicated that SZR plays an essential role to improve sleep in the SZR decoction ( $\mathrm{Li}$ and $\mathrm{Bi}, 2006)$. However, our previous systematic review indicated that the current evidence is insufficient to support the routine use of SZR decoction for insomnia because of poor methodological quality of the included studies (Xie et al., 2013). In addition, only data from systematic reviews of high-quality RCTs will receive 1a-evidence according to the levels of evidence from the Centre of Evidence-Based Medicine in Oxford (Glasziou et al., 2004). Thus, we conduct an updated systematic review of Chinese herbal formulae that contains SZR (FSZR) for insomnia according to the selected high-quality RCTs.

\section{METHODS}

The systematic review and meta-analysis was preformed according to the Preferred Reporting Items in Systematic Reviews and Meta-Analyses (PRISMA) statement (Moher et al., 2010).

\section{Database and Search Strategies}

We have electronically searched Cochrane Central Register of Controlled Trials (CENTRAL), PubMed, Chinese National Knowledge Infrastructure (CNKI), Wangfang database, Chinese Biomedical Database (CBM), EMBASE and VIP Journals Database from inception to November 2016 by using the following key words: (semen ziziphi spinosae OR ziziphus jujuba OR suan zao ren OR suanzaoren) AND (dyssomnia OR insomnia OR sleep OR sleep disorder OR sleep maintenance OR sleep initiation) AND (randomized controlled trial OR randomized clinical trial OR controlled clinical trial) in English or in Chinese. In addition, we further hand-searched the reference lists from related literature.

\section{Eligibility Criteria Types of Studies}

RCTs that evaluated the efficacy and safety of FSZR for insomnia were selected, regardless of language, publication status, or population characteristics. Only RCTs with positive for at least 4 out of 7 for the Cochrane risk of bias domains were included in further analyses. Quasi-randomized trials, for example, allocated by medical record number, date of birth, or the order in which participants are included in the study, were excluded.

\section{Types of Participants}

All participants with a diagnosis of insomnia by using Chinese classification of mental disorders (CCMD) (Chinese Society of Psychiatry, 1989, 1995, 2001), or ICSD (American Sleep Disorders Association, 1990; American Academy of Sleep Medicine, 2005, 2014), or Guideline for Clinical Trials of New Patent Chinese Medicines (Zheng, 1993) were included. Insomnia disorder caused by a co-occurring psychiatric or 
medical condition, or withdrawal from a drug or substance was excluded.

\section{Types of Interventions}

Analyzed interventions in the experimental groups used FSZR, regardless of the dose or the method or the form or the duration. Comparator interventions were given diazepam, placebo or vehicle treatment or basic treatment (i.e., supportive treatment other than diazepam). Studies comparing FSZR with any other TCM agent were excluded.

\section{Types of Outcome Measures}

The primary outcome measures were Polysomnography (PSG) index and Pittsburgh Sleep Quality Index (PSQI) (Buysse et al., 1989 ) at the end of the treatment course. The secondary outcome measures were clinical effective rate and adverse events. Evaluation standards for clinical efficacy based on Guideline for Clinical Trials of New Patent Chinese Medicines (Zheng, 1993) were as follows: (1) clinical recovery: sleep time returned to normal, or the nocturnal sleep time over $6 \mathrm{~h}$, deep sleep, waking up invigorating; (2) markedly effective: significant improvement of sleep, increased at least $3 \mathrm{~h}$ of total sleep time; depth of sleep increased; (3) effective: amelioration in symptoms; increased $<3 \mathrm{~h}$ of total sleep time; (4) ineffective: no significant improvement of sleep, or deteriorated after treatment.

\section{Study Selection and Data Collection}

All studies were searched in the electronic databases independently by two authors. The authors filtered the titles and abstracts of retrieved studies for inclusion. The further review was based on going through the full-text and assessing study eligibility. The exclusion reasons have been recorded. A standardized study extraction was based on first author, population characteristics, study design, study inclusion and exclusion criteria, age and disease duration range of participants, intervention details, duration of treatment, outcomes and follow-up time. For the disagreements, we resolved by discussion between the two authors or hold counsel with the third author.

\section{Risk of Bias in Individual Studies}

The criteria recommended by the Cochrane Collaboration (Higgins and Green, 2011) were used to assess the risk of bias in the included studies. The score ranged from 0 to 7 . Divergences were well settled through consulting with correspondence authors.

\section{Data Synthesis and Analysis}

We performed the statistical analysis on the data by applying the Cochrane Collaboration Review Manager software (RevMan 5.0). Between-study heterogeneity was valued using the chisquare and the $I^{2}$ statistic was calculated. The heterogeneity was expected statistically significant if the $P$-values were $<0.05$. Presence or absence of significant heterogeneity decided the option of random effects model or fixed effects model. Outcome data were calculated using standardized mean difference (SMD) with 95\% confidence interval (CI) for continuous outcomes, and risk ratio (RR) with 95\% CI for dichotomous outcomes. The Grading of Recommendations Assessment, Development and
Evaluation (GRADE) methodology (Schunemann et al., 2013) was used to rate the quality of evidence.

\section{RESULTS}

\section{Study Selection}

The search strategy has retrieved 2,561 studies in total, among which 711 were considered duplicates. Of the remaining 1,850 articles, 983 articles were eliminated because of review or case report, or summary of treating experience or animal studies. For a further step, we excluded 812 studies by reason that they not used FSZR, or used any other TCM, or not real RCTs. Fifty-five RCTs were left and evaluated by the Cochrane risk of bias tool. Among which, 13 studies (Zhou et al., 2002; Lian et al., 2009; Li et al., 2009; Liu and Nan, 2009; Jiang, 2010; Long, 2010; Wang Z. T. et al., 2010; Jing, 2011; Lu, 2011; Pan, 2011; Wang et al., 2013; Yuan et al., 2013; Shi et al., 2014) were assessed in RCTs with a cumulative score of at least 4 out of 7 for the Cochrane risk of bias tool domains and were ultimately included. The screening process is summarized in a flow diagram (Figure 1).

\section{Study Characteristics}

Characteristic of included studies was showed in Table 1. All involved articles were published in Chinese language. All 13 trials were RCTs, and the sample size ranged from 33 to 366, mean aged 26.42 to 71.9 years. The disease durations before treatment lasted from 39 days to 7.9 years. The duration of treatment ranged from 15 days to 12 weeks. Five studies (Zhou et al., 2002; Lian et al., 2009; Jiang, 2010; Yuan et al., 2013; Shi et al., 2014) reported the follow-up time, which lasted from 1 week to 12 weeks. Twelve studies (Lian et al., 2009; Li et al., 2009; Liu and Nan, 2009; Jiang, 2010; Long, 2010; Wang Z. T. et al., 2010; Jing, 2011; Lu, 2011; Pan, 2011; Wang et al., 2013; Yuan et al., 2013; Shi et al., 2014) reported the PSQI score and three studies (Li et al., 2009; Long, 2010; Wang et al., 2013) reported the PSG. The high-frequency herbs in the 13 included articles were detailed in Table 2. The top 6 most frequently used herbs were Spine date seed (Semen Ziziphi Spinosae), Indian buead (Indian buead), tuber fleeceflower stem (Caulis Polygoni Multiflori), debark peony root (Radix Paeoniae Alba), milkwort root (Radix Polygalae), Chinese angelica (Radix Angelicae Sinensis), which were used more than 3 times. The dose of SZR in each FSZR constituents varied from 24 to $36 \mathrm{~g}$ per day (Li et al., 2009; Jiang, 2010; Long, 2010; Pan, 2011). The ingredients and the usage of FSZR formulae were detailed in Table 3. The sleep time was evaluated by Polysomnograghy or electroencephalogram in three studies (Li et al., 2009; Long, 2010; Wang et al., 2013) and PSQI scores in 12 studies (Lian et al., 2009; Li et al., 2009; Liu and Nan, 2009; Jiang, 2010; Long, 2010; Wang Z. T. et al., 2010; Jing, 2011; Lu, 2011; Pan, 2011; Wang et al., 2013; Yuan et al., 2013; Shi et al., 2014). We have detailed the data in the Table 4.

\section{Risk of Bias within Studies}

The Cochrane's risk of bias score of included studies ranged from 4 to 7 (Table 5). All of the retrieved articles belonged to RCT. Among which, 11 out of 13 articles described the method of random sequences generation. Four studies (Lian et al., 2009; 


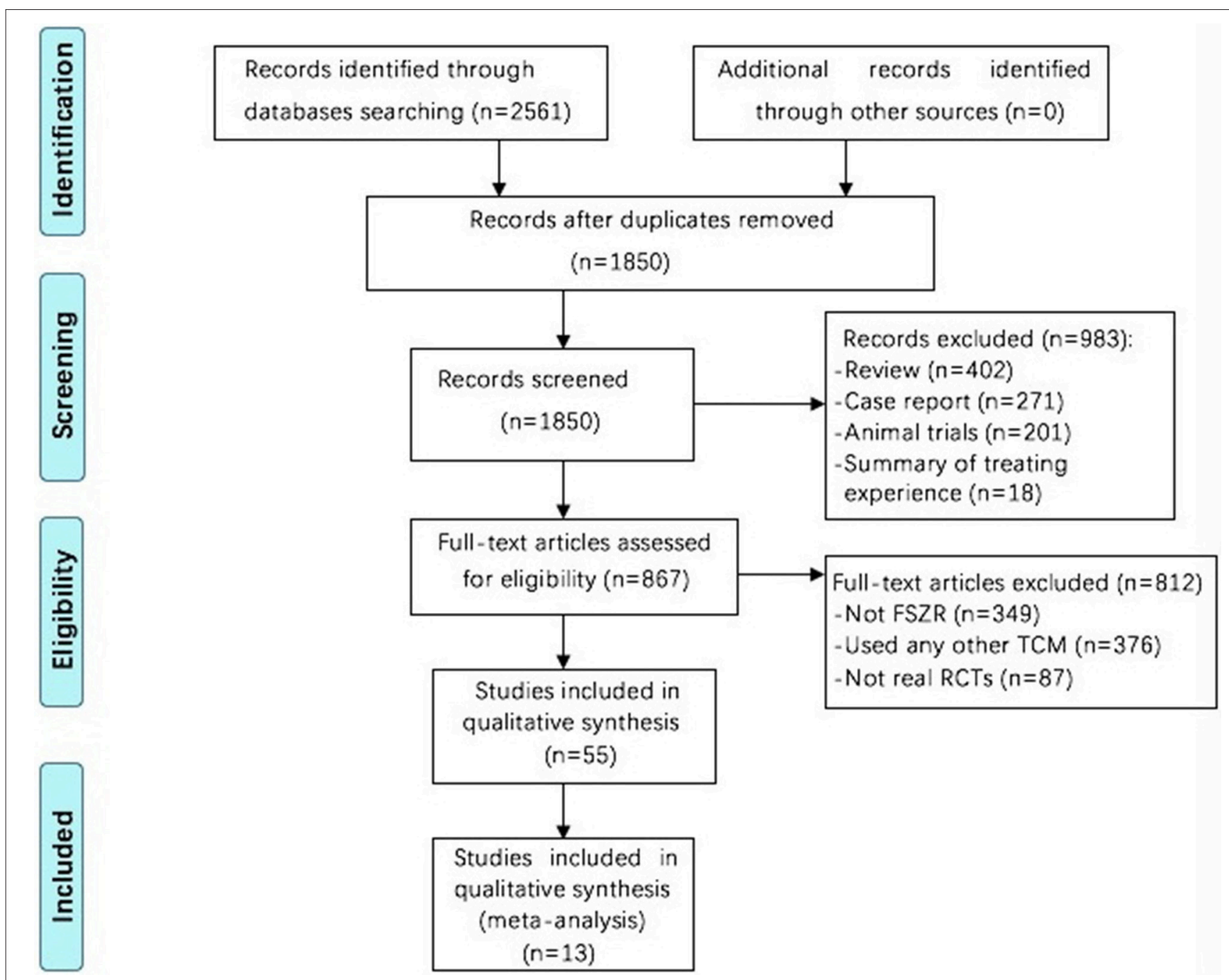

FIGURE 1 | Flowchart of study screening. FSZR, Chinese formulae that contains suanzaoren. TCM, Traditional Chinese medicine; RCTs, randomized controlled trials.

Li et al., 2009; Pan, 2011; Yuan et al., 2013) implemented the concealment allocation. The blinding was used in 11 studies except two studies (Pan, 2011; Shi et al., 2014), including seven double blinding (Zhou et al., 2002; Lian et al., 2009; Li et al., 2009; Wang Z. T. et al., 2010; Jing, 2011; Wang et al., 2013; Yuan et al., 2013) and four single blinding (Liu and Nan, 2009; Jiang, 2010; Long, 2010; Lu, 2011). There were five studies (Lian et al., 2009; Li et al., 2009; Jing, 2011; Wang et al., 2013; Yuan et al., 2013) described the blinding procedure. Three studies (Li et al., 2009; Yuan et al., 2013; Shi et al., 2014) avoided the detection bias by blind the statisticians. Selection bias and other bias were not found in all included studies.

\section{Effectiveness}

\section{FSZR vs. Placebo}

There were six studies (Lian et al., 2009; Liu and Nan, 2009; Wang Z. T. et al., 2010; Jing, 2011; Lu, 2011; Wang et al., 2013) comparing FSZR with placebo for treating insomnia (Table 1). Meta-analysis of above six studies showed significant betweengroup difference in PSQI scores $(n=438, \mathrm{SMD}=-1.05,95 \% \mathrm{CI}$ : -1.49 to $-0.60, P<0.00001$, heterogeneity $\mathrm{X}^{2}=23.36, \mathrm{df}=5$, $\left.P=0.004, I^{2}=74 \%\right)$. After removing study by Lu et al. (Lu, 2011) that was considered the potential sources of the heterogeneity because of small sample size, meta-analysis of five studies (Lian et al., 2009; Liu and Nan, 2009; Wang Z. T. et al., 2010; Jing, 2011; Wang et al., 2013) showed that FSZR better reduce the PSQI score than that of placebo in PSQI scores $(n=390, \mathrm{SMD}=-0.82$, $95 \%$ CI: -1.03 to $-0.61, P<0.00001$, heterogeneity $\mathrm{X}^{2}=6.24$, $\mathrm{df}=4, P=0.18, I^{2}=36 \%$; Figure 2). Meta-analysis of four studies (Lian et al., 2009; Liu and Nan, 2009; Jing, 2011; Lu, 2011) found significant difference in clinical effective rate between the FSZR and placebo groups $(n=256, \mathrm{RR}: 1.73,95 \% \mathrm{CI} 1.25$ to 2.39, $P=0.0009$, heterogeneity $\mathrm{X}^{2}=6.59$, $\mathrm{df}=3, P=0.09$, $I^{2}=54 \%$ ). After removing study by Liu et al. (Liu and Nan, 2009) 
Chou et al.

Suanzaoren Formulae for Insomnia

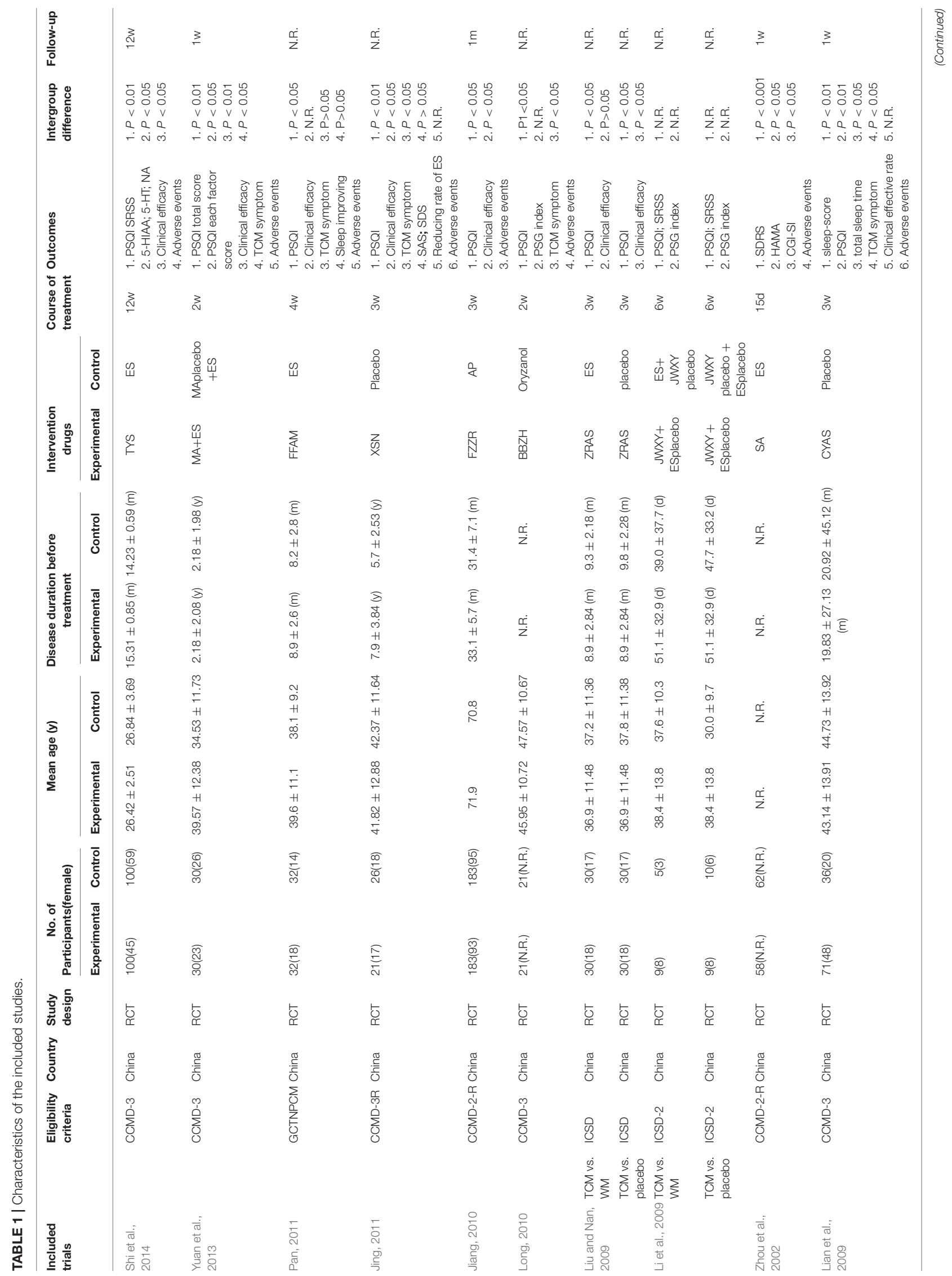

Frontiers in Pharmacology | www.frontiersin.org

5

February 2018 | Volume 9 | Article 76 


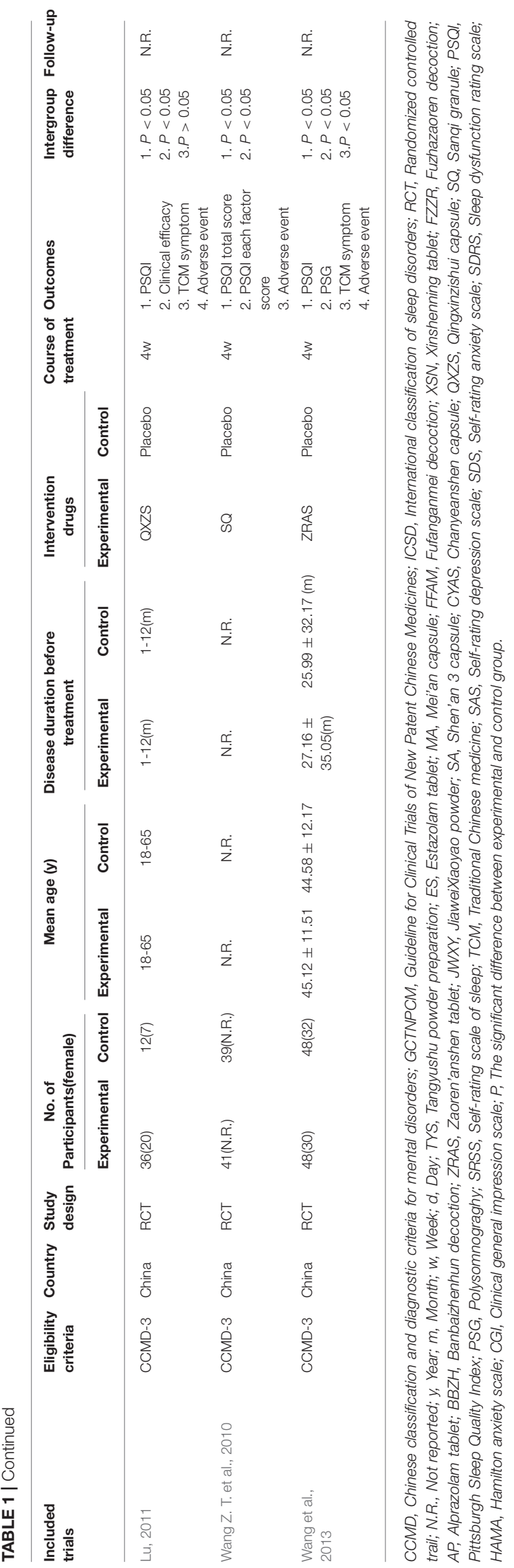

TABLE 2 | Analysis of the high frequency herbs in treatment of insomnia.

\begin{tabular}{llll}
\hline $\begin{array}{l}\text { Chinese } \\
\text { name }\end{array}$ & Common name & Latin name & Frequency \\
\hline Suanzaoren & Spine date seed & Semen ZiziphiSpinosae & 13 \\
Fuling & Indian buead & Indian buead & 4 \\
Shouwuteng & tuber fleeceflower stem & Caulis PolygoniMultiflori & 4 \\
Baishao & debark peony root & Radix Paeoniae Alba & 4 \\
Yuanzhi & milkwort root & Radix Polygalae & 3 \\
Danggui & Chinese angelica & Radix AngelicaeSinensis & 3 \\
Shichangpu & grassleafsweetflag rhizome & RhizomaAcoriTatarinowii & 2 \\
Baihe & lily bulb & BulbusLilii & 2 \\
Chaihu & Chinese thorowax root & Radix Bupleuri & 2 \\
Huanglian & golden thread & RhizomaCoptidis & 2 \\
Zhenzhumu & nacre & Concha Margaritifera & 2 \\
Banxia & pinellia tuber & RhizomaPinelliae & 2 \\
Longchi & Dragon's Teeth & Mastodifossiliadentis & 2 \\
Chenpi & dried tangerine peel & PericarpiumCitriReticulatae & 2 \\
\hline
\end{tabular}

whose diagnostic criteria differed from other three studies, metaanalysis of three studies (Lian et al., 2009; Jing, 2011; Lu, 2011) showed that FSZR was significantly more effective than that of placebo in clinical effective rate $(n=196, \mathrm{RR}: 2.04,95 \%$ CI 1.52 to $2.74, P<0.00001$, heterogeneity $\mathrm{X}^{2}=0.14, \mathrm{df}=2, P=0.93$, $I^{2}=0 \%$; Figure 3 ).

\section{FSZR vs. Diazepam}

Five studies (Zhou et al., 2002; Liu and Nan, 2009; Jiang, 2010; Pan, 2011; Shi et al., 2014) investigated FSZR vs. Diazepam. The meta-analysis was not conducted because of high heterogeneity. Three studies (Jiang, 2010; Pan, 2011; Shi et al., 2014) found that FSZR was more effective than Diazepam in PSQI score $(P>0.05)$, whereas one study (Liu and Nan, 2009) showed no significant difference. FSZR was significantly improving clinical effective rate in two studies (Jiang, 2010; Shi et al., 2014) $(P<0.05)$, but not in other two studies (Liu and Nan, 2009; Pan, 2011) $(P>0.05)$ relative to Diazepam.

\section{FSZR Plus Diazepam vs. FSZR Placebo Plus Diazepam}

Two studies (Li et al., 2009; Yuan et al., 2013) compared FSZR plus Diazepam with FSZR placebo plus Diazepam. Metaanalysis showed a significant reduction in PSQI scores for the combination therapy relative to estazolam alone $(n=74$, $\mathrm{SMD}=-0.53,95 \% \mathrm{CI}:-1.00$ to $-0.06, P=0.03$, heterogeneity $\mathrm{X}^{2}=1.87, \mathrm{df}=1, P=0.17, I^{2}=47 \%$ ) (Figure 4). The study by Yuan et al. (2013) found FSZR was significantly more effective than placebo in clinical effective rate $(P<0.05)$.

\section{Adverse Events}

Twelve out of 13 studies reported adverse events. One study (Shi et al., 2014) reported adverse events in both experimental group and control group, including acratia, somnolence, dizzy, diarrhea, and dry mouth. Two studies (Zhou et al., 2002; Jiang, 
TABLE 3 | Ingredients and usage of FSZR formulae.

\begin{tabular}{|c|c|c|c|c|}
\hline Included studies & Prescription & Constitution & Usage & Preparations \\
\hline Shi et al., 2014 & TYS & $\begin{array}{l}\text { Semen ZiziphiSpinosae, RhizomaCoptidis, PericarpiumCitriReticulatae, Caulis Bambusae in } \\
\text { Taenia, RhizomaAcoriTatarinowii, Radix Polygalaea }\end{array}$ & $15 \mathrm{~g}$ tidpo & Power \\
\hline Yuan et al., 2013 & $M A+E S$ & $\begin{array}{l}\text { Semen ZiziphiSpinosae, Radix Ginseng, Eleutherococcussenticosus, Indian buead, Radix } \\
\text { AngelicaeSinensis, RhizomaLigusticiChuanxionga }\end{array}$ & $4 \mathrm{~g}$ qnpo & Granule \\
\hline Pan, 2011 & FFAM & $\begin{array}{l}\text { Semen ZiziphiSpinosae } 12 \mathrm{~g} \text {, OsDraconis } 9 \mathrm{~g} \text {, Concha Margaritifera } 9 \mathrm{~g} \text {, Caulis } \\
\text { PolygoniMultiflori } 12 \mathrm{~g} \text {, FructusSchisandraeChinensis } 3 \mathrm{~g} \text {, Radix Paeoniae Alba } 9 \mathrm{~g} \text {, Radix } \\
\text { RehmanniaeRecens } 9 \mathrm{~g}\end{array}$ & One dose bid po & Decoction \\
\hline Jing, 2011 & XSN & $\begin{array}{l}\text { Semen ZiziphiSpinosae, Indian buead, Caulis PolygoniMultiflori, Massa MedicataFermentata, } \\
\text { FructusGardeniaea }\end{array}$ & $4 \mathrm{~g}$ tidpo & Tablet \\
\hline Jiang, 2010 & $\mathrm{FZZR}$ & Semen ZiziphiSpinosae $15 \mathrm{~g}$, Indian buead $12 \mathrm{~g}$, FructusCrataegi $15 \mathrm{~g}$ & One dose bid po & Decoction \\
\hline Long, 2010 & $\mathrm{BBZH}$ & $\begin{array}{l}\text { Semen ZiziphiSpinosae } 30 \mathrm{~g} \text {, RhizomaPinelliae } 10 \mathrm{~g} \text {, BulbusLilii30 g, Radix Curcumae } 10 \mathrm{~g} \text {, } \\
\text { Caulis PolygoniMultiflori } 30 \mathrm{~g} \text {, Magnetitum } 30 \mathrm{~g} \text {, Concha Ostreae } 30 \mathrm{~g} \text {, Mastodifossiliadentis } \\
30 \mathrm{~g} \text {, Concha Margaritifera } 30 \mathrm{~g} \text {, Indian buead } 15 \mathrm{~g} \text {, PericarpiumCitriReticulatae } 10 \mathrm{~g} \text {, } \\
\text { RhizomaAcoriTatarinowii } 10 \mathrm{~g}\end{array}$ & Half dose bid po & Decoction \\
\hline Liu and Nan, 2009 & ZRAS & Semen ZiziphiSpinosae, Radix SalviaeMiltiorrhizae, FructusSchisandraeChinensisa & 5 \# qnpo & Tablet \\
\hline Li et al., 2009 & JWXY & $\begin{array}{l}\text { Semen ZiziphiSpinosae } 18 \mathrm{~g} \text {, Poria cum Radix Pini } 15 \mathrm{~g} \text {, Radix Bupleuri } 9 \mathrm{~g} \text {, Radix } \\
\text { AngelicaeSinensis } 10 \mathrm{~g} \text {, Radix Paeoniae Alba } 15 \mathrm{~g} \text {, RhizomaAtractylodisMacrocephalae } 12 \mathrm{~g} \text {, } \\
\text { Radix Glycyrrhizae } 5 \mathrm{~g} \text {, HerbaMenthae } 5 \mathrm{~g} \text {, RhizomaZingiberisRecens } 4 \mathrm{~g}\end{array}$ & $250 \mathrm{ml}$ bid po & Power \\
\hline Zhou et al., 2002 & SA & $\begin{array}{l}\text { Ganoderma, BulbusLilii, Radix PaeoniaeRubra, Radix SalviaeMiltiorrhizae, Cortex } \\
\text { MoutanRadicis, Semen ZiziphiSpinosae, Radix Pseudostellariae, Mastodifossiliadentis, } \\
\text { Succinuma }\end{array}$ & 5 \# qnpo & Capsule \\
\hline Lian et al., 2009 & CYAS & $\begin{array}{l}\text { Semen ZiziphiSpinosae, Periostracum Cicadae, Caulis PolygoniMultiflori, BombyxBatryticatus, } \\
\text { Lumbricus, Radix Paeoniae Alba, RamulusUncariae Cum Uncis, RhizomaPinelliae, Radix } \\
\text { Polygalaea }\end{array}$ & 5\# qnpo & Capsule \\
\hline Lu, 2011 & QXZS & $\begin{array}{l}\text { Semen ZiziphiSpinosaea, RhizomaCoptidis, Radix Scutellariae, Radix Paeoniae Alba, } \\
\text { CollaCoriiAsini, Cortex Cinnamomi, Cortex Albiziae }\end{array}$ & 5 \# tidpo & Capsule \\
\hline $\begin{array}{l}\text { Wang Z. T. et al., } \\
2010\end{array}$ & $\mathrm{SQ}$ & Semen ZiziphiSpinosae, Caulis Spatholobi, Radix Notoginseng, Cirsiumjaponicuma & One dose qdpo & Granule \\
\hline Wang et al., 2013 & ZRAS & $\begin{array}{l}\text { Semen ZiziphiSpinosae, Caulis PolygoniMultiflori, Fructus Mori, FlosAlbiziae, Semen Platycladi, } \\
\text { Radix AngelicaeSinensis, Radix RehmanniaePreparata, Radix Polygalae, Radix Bupleuria }\end{array}$ & 4 \# tidpo & Tablet \\
\hline
\end{tabular}

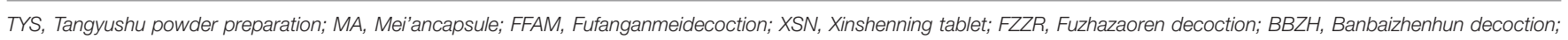

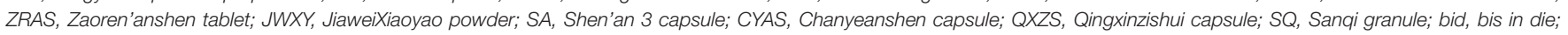
d:day; po, peros; qd, quaquedie; tid, ter in die; w, week; \#, tablet.

TABLE 4 | Characteristics of the Sleeping time of included studies.

\begin{tabular}{|c|c|c|c|c|c|c|}
\hline \multirow[t]{2}{*}{ Included trials } & & \multirow[t]{2}{*}{ Methods } & \multicolumn{2}{|c|}{ Sleeping-time (before) } & \multicolumn{2}{|c|}{ Sleeping-time (after) } \\
\hline & & & Experimental & Control & Experimental & Control \\
\hline Shi et al., 2014 & & PSQI & $N G$ & $N G$ & $N G$ & $N G$ \\
\hline Yuan et al., 2013 & & PSQI & $1.53 \pm 1.07$ & $1.53 \pm 0.97$ & $1.10 \pm 0.88$ & $1.33 \pm 0.96$ \\
\hline Pan, 2011 & & PSQI & $2.58 \pm 1.01$ & $2.46 \pm 0.93$ & $1.05 \pm 0.99$ & $1.71 \pm 0.86$ \\
\hline Jing, 2011 & & PSQI & $N G$ & $N G$ & $N G$ & $N G$ \\
\hline Jiang, 2010 & & PSQI & $2.7 \pm 0.5$ & $2.7 \pm 0.7$ & $0.6 \pm 0.7$ & $1.2 \pm 0.7$ \\
\hline Long, 2010 & & PSG(m) & $360.60 \pm 32.975$ & $355.76 \pm 30.363$ & $368.07 \pm 32.794$ & $358.71 \pm 34.229$ \\
\hline \multirow[t]{2}{*}{ Liu and Nan, 2009} & TCM vs. WM & PSQI & $N G$ & $N G$ & $N G$ & $N G$ \\
\hline & TCM vs. placebo & PSQI & $N G$ & $N G$ & $N G$ & $N G$ \\
\hline \multirow{2}{*}{ Li et al., 2009} & TCM vs. WM & PSG(m) PSQI & $367.5 \pm 52.0 \mathrm{NG}$ & $405.7 \pm 38.2 \mathrm{NG}$ & $391.8 \pm 44.5 \mathrm{NG}$ & $378.6 \pm 53.9 \mathrm{NG}$ \\
\hline & TCM vs. placebo & PSG(m) PSQI & $367.5 \pm 52.0 \mathrm{NG}$ & $385.8 \pm 43.4 \mathrm{NG}$ & $391.8 \pm 44.5 \mathrm{NG}$ & $405.6 \pm 49.4 \mathrm{NG}$ \\
\hline Zhou et al., 2002 & & PSQI & $N G$ & $N G$ & $N G$ & $N G$ \\
\hline Lian et al., 2009 & & PSQI & $4.91 \pm 0.87$ & $4.55 \pm 1.06$ & $6.29 \pm 1.17$ & $5.22 \pm 1.16$ \\
\hline Lu, 2011 & & PSQI & $2.39 \pm 0.68$ & $2.49 \pm 0.69$ & $1.12 \pm 0.67$ & $2.32 \pm 0.72$ \\
\hline Wang Z. T. et al., 2010 & & PSQI & $4.32 \pm 1.56$ & $4.18 \pm 1.81$ & $5.77 \pm 1.47$ & $4.87 \pm 1.99$ \\
\hline Wang et al., 2013 & & PSG(m) PSQI & $339.66 \pm 77.1 \mathrm{NG}$ & $368.90 \pm 70.12 \mathrm{NG}$ & $388.26 \pm 74 \mathrm{NG}$ & $367.93 \pm 86.07 \mathrm{NG}$ \\
\hline
\end{tabular}

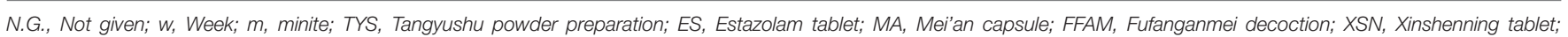

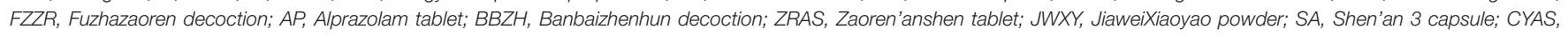

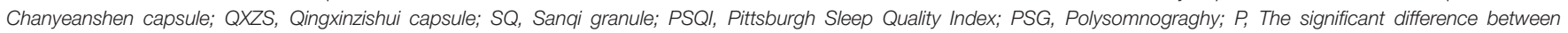
experimental and control group. 
2010) reported side effects in control group alone, including dry mouth, constipation, nausea, somnolence, dizzy, fatigued, and memory decline. Serious and life-threatening adverse events, such as an irregular heartbeat, seizures, and death were not happened in all included studies.

\section{Mechanisms of SZR for Insomnia}

SZR exerts a range of sedative and hypnotic actions mediated primarily by the GABAergic and serotonergic system (Ma

TABLE 5 | The methodological quality of included studies.

\begin{tabular}{lllllllll}
\hline & A & B & C & D & E & F & G & Total score \\
\hline Shi et al., 2014 & + & $?$ & $?$ & + & + & + & + & $5+$ \\
Yuan et al., 2013 & + & + & + & + & + & + & + & $7+$ \\
Pan, 2011 & + & + & $?$ & + & $?$ & + & + & $5+$ \\
Jing, 2011 & + & $?$ & + & + & $?$ & + & + & $5+$ \\
Jiang, 2010 & + & $?$ & + & + & $?$ & + & + & $5+$ \\
Long, 2010 & + & $?$ & + & + & $?$ & + & + & $5+$ \\
Liu and Nan, 2009 & + & $?$ & + & + & $?$ & + & + & $5+$ \\
Li et al., 2009 & + & + & + & + & + & + & + & $7+$ \\
Zhou et al., 2002 & + & $?$ & + & + & $?$ & + & + & $5+$ \\
Lian et al., 2009 & + & + & + & + & $?$ & + & + & $6+$ \\
Lu, 2011 & $?$ & $?$ & + & + & $?$ & + & + & $4+$ \\
Wang Z. T. et al., 2010 & + & $?$ & + & + & $?$ & + & + & $5+$ \\
Wang et al., 2013 & $?$ & $?$ & + & + & $?$ & + & + & $4+$ \\
\hline
\end{tabular}

$A$, random sequence generation; $B$, allocation concealment; $C$, blinding of participants and personnel; $D$, blinding of outcome assessment; $E$, imcomplete outcome data; $F$, selective reporting; $G$, other sources of bias. et al., 2007; Wang L. E. et al., 2010; Shergis et al., 2017) (Figure 5). The SZR typically contains complex mixtures of phytochemicals, including sanjoinine A, Jujuboside A (JuA), spinosin and other flavonoids (Yang et al., 2013). Sanjoinine A, one of the aporphine alkaloid from SZR, was demonstrated to enhance sleep behaviors and augment pentobarbital-induced sleeping behaviors through GABAergic system (Han et al., 2009; Rodríguez and Rodríguez, 2017). Furthermore, Sanjoinine A increases chloride influx and GABA synthesis via glutamic acid decarboxylase (GAD 65/67) activation in cultured cerebellar granule cells, inducing prolonged sleeping time (Zhang et al., 2003). JuA inhibits the rat hippocampus excitatory state in vivo and in vitro through glutamate-mediated excitatory signal pathway (Cao et al., 2010), affecting GABAergic and serotonergic system. Low dose of JuA exerts sedative-hypnotic effects related to increasing the GABAA receptor $(\alpha 1, \alpha 5, \beta 2)$ gene expression (You et al., 2010). Flavonoids in the water extract of SZR also contribute to insomnia treatment. The flavonoid 6-hydroxyflavone is involved in the binding of GABAA receptors showing partial agonistic action (Ren et al., 2010). The administration of spinosin, a C-glycoside flavonoid, showed significant sedative effects at increasing total sleep time and reducing sleep latency in pentobarbital treated rat group (Lee et al., 2016).

\section{The Quality of the Evidence}

Summary of Findings (SOF) tables were provided by the GRADE profiler. The quality of the evidence was high or moderate according to the GRADE assessment, we summarized the result in Table 6.

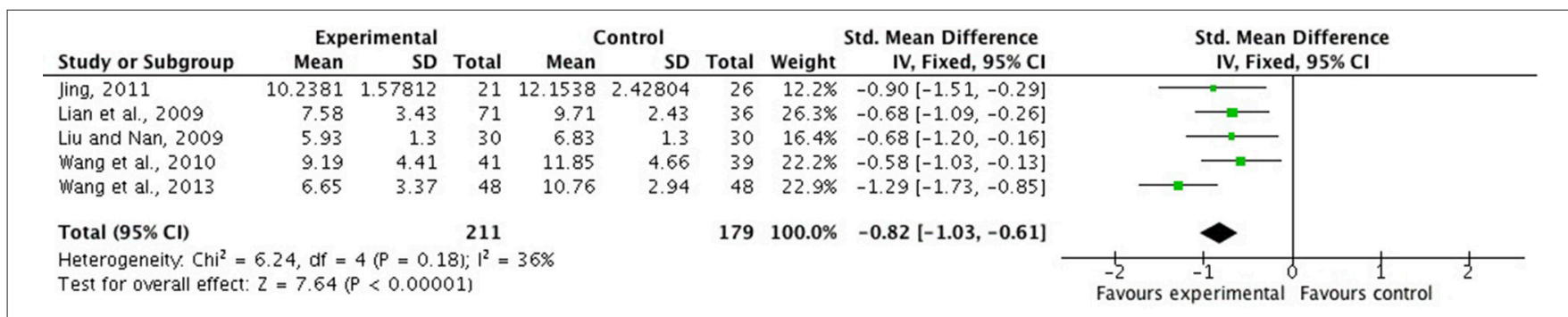

FIGURE 2 | PSQI scores of FSZR vs. placebo. PSQI, Pittsburgh Sleep Quality Index; FSZR, Chinese formulae that contains suanzaoren.

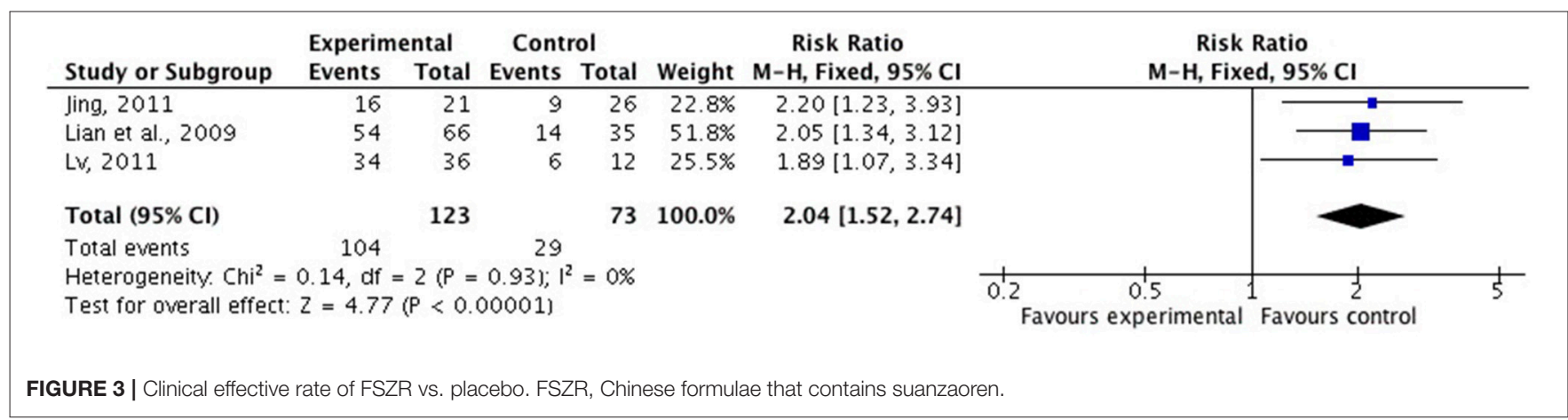




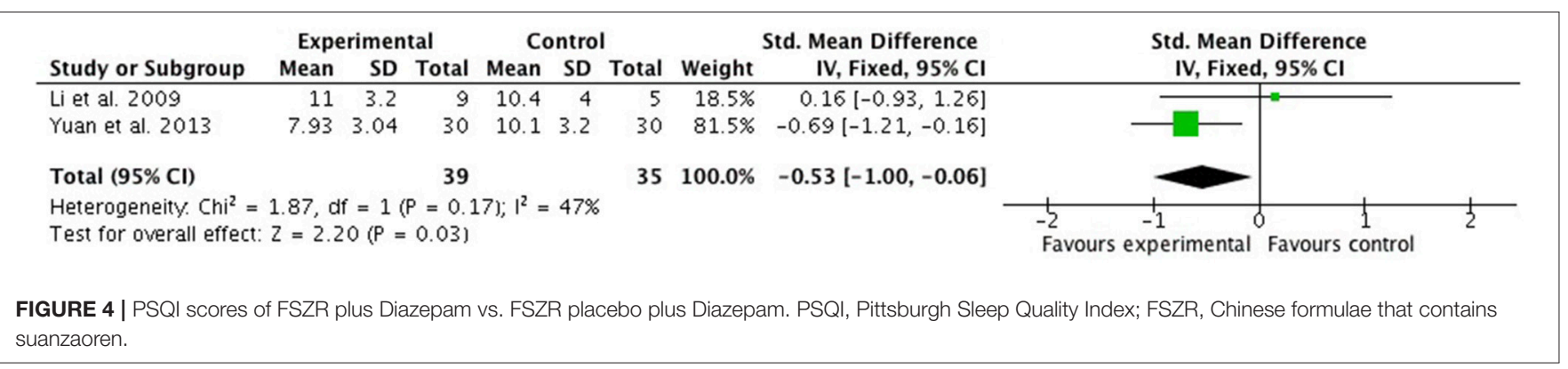

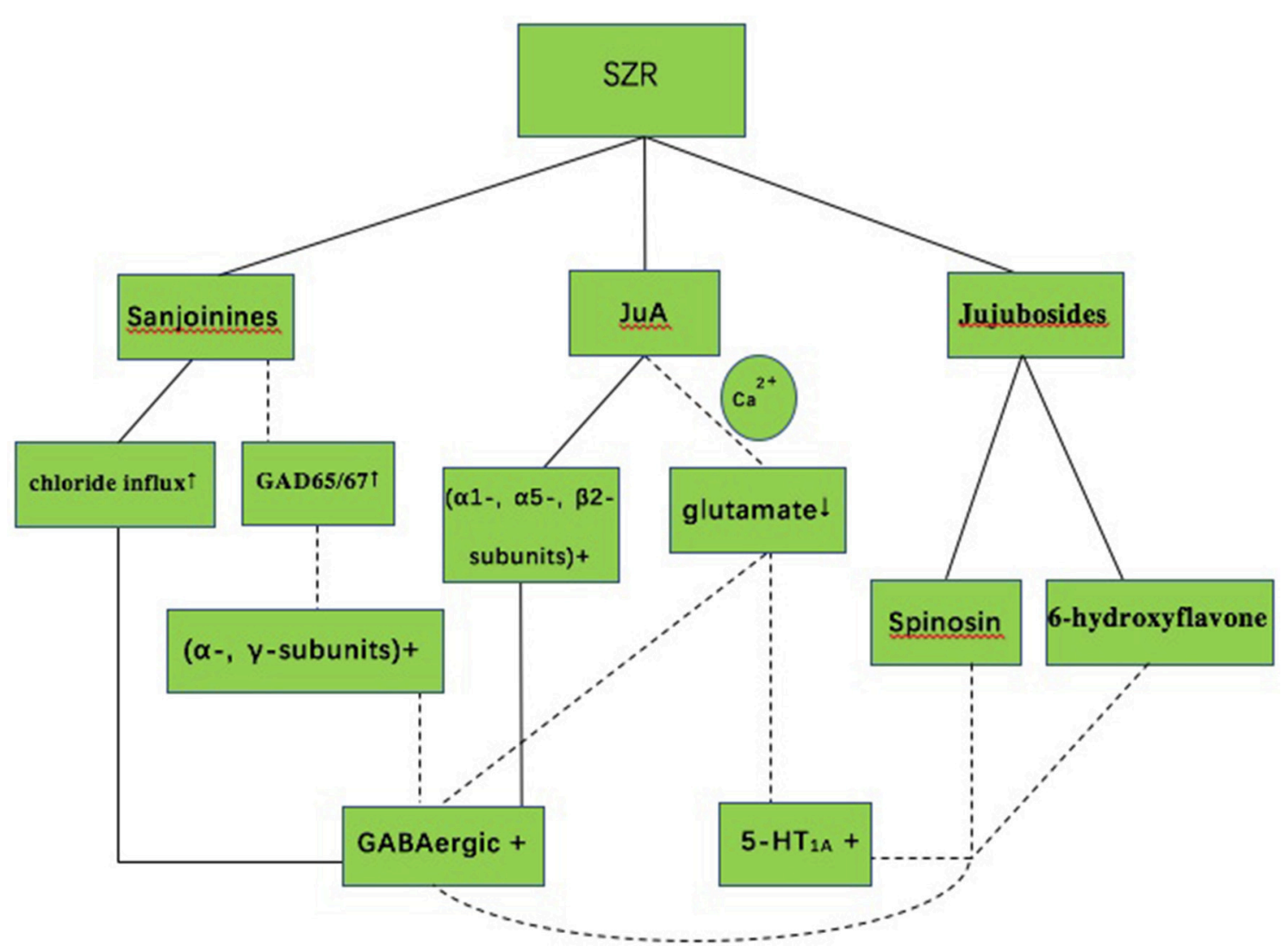

FIGURE 5 | The mechanism of sedative-hypnotic effect in SZR for insomnia. SZR, suanzaoren; GABA, gamma-aminobutyric acid; 5-HT 1 A, 5-hydroxytryptamine (1A); JuA, Jujuboside A; Solid lines indicate established effects, whereas dashed lines represent putative mechanism.

TABLE 6 | Quality of evidence by GRADE system.

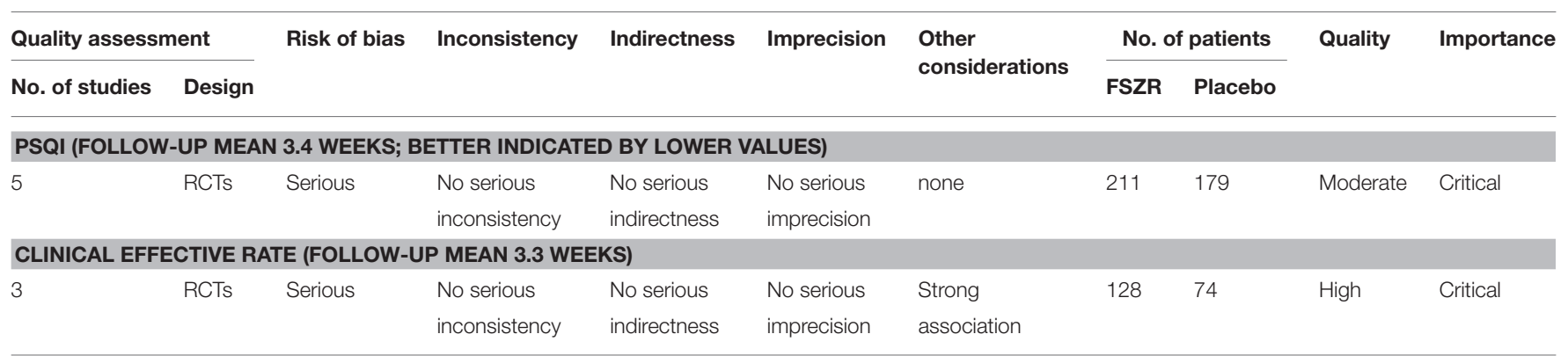

RCT: randomized controlled trial; PSQI, Pittsburgh Sleep Quality Index; FSZR, Chinese formulaethat contains Suanzaoren. 


\section{DISCUSSION}

\section{Summary of Main Findings}

Our previous system review (Xie et al., 2013) showed that the current evidence was insufficient to support the efficacy and safety of SZRD for insomnia due to lack of high-quality RCTs. The present study is an updated systematic review based on the high-quality RCTs. Thirteen studies with 1,454 individuals were identified in analysis. The findings demonstrated that FSZR used as a monotherapy was superior to placebo and as an adjunct therapy was superior to Diazepam alone in terms of PSQI score and clinical effective rate, whereas there were mixed results comparing FSZR with Diazepam directly. There were fewer adverse effects in comparison with controls and no lifethreatening side effects were happened in all included studies. The quality of the evidence was high or moderate based on the updated GRADE methodology and profiler.

\section{LIMITATIONS}

The strength of this study was that all studies included had low risk of bias with positive for at least four out of seven for the Cochrane risk of bias domains. However, we did acknowledge that there were some methodological limitations which were worth noting. Firstly, allocation concealment was used in two studies (Ma et al., 2007; Moher et al., 2010). The trials with inadequate or unclear concealment of allocation were about $18 \%$ more "beneficial" than that with adequate concealment (95\% CI 5\% to 29\%; Higgins and Green, 2011). Secondly, blinding was an essential method to limit the occurrence of performance bias and ascertainment bias in clinical trials (Health Canada, 2003). Although there were 11 out of 13 studies that mentioned the blinding, double-blinding and placebo design was reported in six studies (Lian et al., 2009; Wang Z. T. et al., 2010; Jing, 2011; Lu, 2011; Wang et al., 2013; Yuan et al., 2013). One of the main reasons for not using double-blinded study was that the placebos of CHMs is difficult to prepared in the same color, flavor and taste. Thirdly, the follow-up period was not long enough to achieve crucial results because insomnia may wax and wane with or without treatment. Thus, a longer follow-up period was necessary (Xie et al., 2013). At least 6-month follow-up may assess whether a sustained effect and safety of FSZR for insomnia can persist for a long period (European Medicines Agency, 2011). Finally, the quality control of herbal preparations is crucial for the validity of the study results. However, only some patent FSZR mentioned in this important issue. Suanzaoren contains complex mixtures of phytochemicals such as sanjoinine A, Jujuboside A, spinosin, and other flavonoids, which can be further used for the marker of quality control of the herbal preparations.

\section{Implication for Practice}

This is an updated system review of high-quality RCTs to assess the efficacy and safety of FSZR for insomnia. In the present study, patients receiving FSZR monotherapy was superior to placebo, and equivalent to Diazepam. Combined FSZR with Diazepam was superior to Diazepam alone. Furthermore, FSZR caused fewer side effects than that of Diazepam. Therefore, FSZR therapy may be effective and well tolerated for the treatment of insomnia. Taken together, our findings supported that clinicians may option FSZR as an alternative treatment for insomnia.

\section{Implication for Research}

There are many implications arising from research. First, although double-blinding is encouraged in RCTs, it is inherently difficult in herbal placebo because of the specific color, flavor, and taste of CHMs (Ni et al., 2015). One of possible solutions is that herbs can be prepared as tablets or capsules to facilitate the development of a convincing placebo ( $\mathrm{Fu}$ et al., 2013); however, the use of capsules is still challenged because of the change of efficacy and characteristics of drugs (Stegemann and Bornem, 1999). Second, SZR is the most frequently used herb for insomnia, and it is regarded as an essential constitute in numbers of classical herbal formulae ( $\mathrm{Li}$ and Bi, 2006; Lei et al., 2015). In the present study, the most frequently used herbs were Semen Ziziphi Spinosae, Indian buead, Caulis Polygoni Multiflori, Radix Paeoniae Alba, Radix Polygalae, Radix Angelicae Sinensis, which is worth further carrying out rigor RCTs as candidate formula. Third, Sanjoinine A, JuA, and flavonoids in the water extract of SZR contributed to its sedative-hypnotic effects. The increasing of chloride influx and over-expression of $\alpha$ - and $\gamma$-subunit GABA receptor was involved in the mechanisms of these effects. The modification of serotonin and glutamate also inducing sleep, but the exact role of the regulation of sleep is still unknown. Further experimental studies are required to unravel the mechanisms of FSZR for insomnia.

\section{CONCLUSION}

In the present study, the findings demonstrated that FSZR therapy was effective and well tolerated for insomnia through sedative and hypnotic actions primarily mediated by the GABAergic and serotonergic system. Thus, FSZR could be an alternative treatment for insomnia in clinical practice.

\section{AUTHOR CONTRIBUTIONS}

G-QZ and YL: contribute as the senior authors and the principal investigator (PI) of this study; Q-HZ, X-LZ, and M-BX: write the first draft of the manuscript and contribute to the overall design; G-QZ and YL: refine the study; T-YJ and P-QR: identified, reviewed studies for eligibility and performed the meta-analysis of data; All authors read, critically reviewed and approved the final manuscript.

\section{ACKNOWLEDGMENTS}

This project was supported by the Young and Middle-Aged University Discipline Leaders of Zhejiang Province, China (2013277); Zhejiang Provincial Program for the Cultivation of High-level Health talents (2015). 


\section{REFERENCES}

American Academy of Sleep Medicine (2005). The International Classification of Sleep Disorders: Diagnostic and Coding Manual. 2nd Edn. Westchester, IL: American Academy of Sleep Medicine.

American Academy of Sleep Medicine (2014). International Classification of Sleep Disorders 3rd Edn. Darien, IL: American Academy of Sleep Medicine.

American Sleep Disorders Association (1990). International Classification of Sleep Disorders: Diagnostic and Coding Manual. American Sleep Disorders Association.

Asnis, G. M., Thomas, M., and Henderson, M. A. (2015). Pharmacotherapy treatment options for insomnia: a primer for clinicians. Int. J. Mol. Sci. 17:50. doi: 10.3390/ijms17010050

Baglioni, C., Battagliese, G., Feige, B., Spiegelhalder, K., Nissen, C., Voderholzer, U., et al. (2011). Insomnia as a predictor of depression: a meta-analytic evaluation of longitudinal epidemiological studies. J. Affect Disord. 135, 10-19. doi: 10.1016/j.jad.2011.01.011

Buysse, D. J. (2013). Insomnia. JAMA 309, 706-716. doi: 10.1001/jama.2013.193

Buysse, D. J., Rd, R. C., Monk, T. H., Berman, S. R., and Kupfer, D. J. (1989). The pittsburgh sleep quality index: a new instrument for psychiatric practice and research. Psychiatry Res. 28, 193-213. doi: 10.1016/0165-1781(89) $90047-4$

Cao, J. X., Zhang, Q. Y., Cui, S. Y., Cui, X. Y., Zhang, J., Zhang, Y. H., et al. (2010). Hypnotic effect of jujubosides from Semen Ziziphi Spinosae. J. Ethnopharmacol. 130, 163-166. doi: 10.1016/j.jep.2010.03.023

Chen, L. C., Chen, I. C., Wang, B. R., and Shao, C. H. (2009). Drug-use pattern of Chinese herbal medicines in insomnia: a 4-year survey in Taiwan. J. Clin. Pharm. Ther. 34, 555-560. doi: 10.1111/j.1365-2710.2009.01038.x

Chinese Society of Psychiatry (1989). The Chinese Classification and Diagnostic Criteria of Mental Disorders Version 2 (CCMD-2). Changsha: Institute of Mental Health, Hunan Medical University.

Chinese Society of Psychiatry (1995). The Chinese Classification and Diagnostic Criteria of Mental Disorders Version 2 Revision (CCMD-2R). Nanjing: Southeast University Press.

Chinese Society of Psychiatry (2001). The Chinese Classification and Diagnostic Criteria of Mental Disorders Version 3 (CCMD-3). Jinan: Chinese Society of Psychiatry.

Daley, M., Morin, C. M., Leblanc, M., Grégoire, J. P., and Savard, J. (2009). The economic burden of insomnia: Direct and indirect costs for individuals with insomnia syndrome, insomnia symptoms, and good sleepers. Sleep 32, 55-64. doi: 10.1080/00365510510013884

European Medicines Agency (2011). Committee for Medical Product of Human Use. Guideline on medicinal products for the treatment of Insomnia[EB/OL]. Available online at: http://www.ema.europa.eu/ema/index.jsp?curl=pages/ regulation/general/general_content_001178.jsp\&mid=WC0b01ac0580034cf5 (Accessed September 1, 2011).

Fan, T. P., Yeh, J. C., Leung, K. W., Yue, P. Y. K., and Wong, R. N. S. (2006). Angiogenesis: from plants to blood vessels. Trends Pharmacol. Sci. 27, 297-309. doi: 10.1016/j.tips.2006.04.006

Fernandez-Mendoza, J., and Vgontzas, A. N. (2013). Insomnia and its impact on physical and mental health. Curr. Psychiatry Rep. 15:418. doi: 10.1007/s11920-013-0418-8

Frass, M., Strassl, R. P., Friehs, H., Müllner, M., Kundi, M., and Kaye, A. D. (2012). Use and acceptance of complementary and alternative medicine among the general population and medical personnel: a systematic review. Ochsner J. 12, 45-56. doi: 10.1043/1524-5012-12.1.45

Fu, D. L., Lu, L., Zhu, W., Li, J. H., Li, H. Q., Liu, A. J., et al. (2013). Xiaoxuming decoction for acute ischemic stroke: a systematic review and meta-analysis. $J$. Ethnopharmacol. 148, 1-13. doi: 10.1016/j.jep.2013.04.002

Glasziou, P., Vandenbroucke, J., and Chalmers, I. (2004). Assessing the quality of research. BMJ 328, 39-41. doi: 10.1136/bmj.328.7430.39

Gu, G. G. (2007). Shennong's Materia Medica Classic. Beijing: Academy Press.

Han, H., Ma, Y., Eun, J. S., Li, R., Hong, J. T., Lee, M. K., et al. (2009). Anxiolytic-like effects of sanjoinine A isolated from Zizyphi Spinosi Semen: possible involvement of GABAergic transmission. Pharmacol. Biochem. Behav. 92, 206-213. doi: 10.1016/j.pbb.2008.11.012

Health Canada (2003). Guidance for Industry Statistical Principles for Clinical Trials ICH Topic E9. Available onlne at: http://www.hc-sc.gc.ca/dhp-mps/ prodpharma/applic-demande/guide-ld/ich/efficac/e9-eng.php\#fnb1-ref (Accessed November 6, 2015).

Higgins, J. P., and Green, S. (2011). Cochrane Handbook for Systematic Reviews of Interventions Version 5.1.0. The Cochrane Collaboration. Available online at: http://handbook.cochrane.org/ (Accessed 31 November, 2016).

Huang, J., Zhang, C., Zhao, X., Fei, Z., Wan, K., Zhang, Z., et al. (2016). The jujube genome provides insights into genome evolution and the domestication of sweetness/acidity taste in fruit trees. PLoS Genet. 12:3e10063. doi: 10.1371/journal.pgen.1006433

Jiang, H. Q. (2010). Observation on effect of fu zha zao ren decoction for senile chronic insomnia. Med. Info. 23:189. doi: 10.3969/j.issn.1672-2779.2010.15.017

Jiang, J. G., Huang, X. J., and Chen, J. (2007). Separation and purification of saponins from Semen Ziziphus jujuba and their sedative and hypnotic effects. J. Pharm. Pharmacol. 59, 1175-1180. doi: 10.1211/jpp.59.8.0017

Jing, X. W. (2011). Randomized and Controlled on the Treatment of Xin-shen-ning for Insomnia Patients with Heart-Spleen Deficiency and Internal Disturbance of Deficient-Heat: 48 Cases. dissertation, Chengdu University of Chinese Medicine, Chendu.

Krystal, A. (2009). Sleep Disorders: Neuropharmacology, 2nd Edn. Illinois: Sleep Research Society.

Lee, Y., Jeon, S. J., Lee, H. E., Jung, I. H., Jo, Y. W., Lee, S., et al. (2016). Spinosin, a C-glycoside flavonoid, enhances cognitive performance and adult hippocampal neurogenesis in mice. Pharmacol. Biochem. Behav. 145, 9-16. doi: $10.1016 /$ j.pbb.2016.03.007

Leger, D., and Bayon, V. (2010). Societal costs of insomnia. Sleep Med. Rev. 14, 379-389. doi: 10.1016/j.smrv.2010.01.003

Lei, L., Wen, X. R., Yang, S., Yuan-Bai, L. I., Xing, Y. H., Chu, J. N., et al. (2015). Study on traditional Chinese medicine prescriptions for curing insomnia based on association rules mining. Chin. J. Libr. Inf. Sci. Tradit. Chin. Med. 39, 16-19. doi: 10.3969/j.issn.2095-5707.2015.01.004

Li, J. (2006). Formulas of Traditional Chinese Medicine. Beijing: Higher Education Press.

Li, J., and Deng, T. (1995). Great Dictionary of Chinese medicine. Beijing: People's Medical Publishing House.

Li, Y. J., and Bi, K. S. (2006). Study on the therapeutic material basis of traditional Chinese medicinal preparation Suanzaoren decoction. Chem. Pharm. Bull. 54, 847-851. doi: 10.1248/cpb.54.847

Li, Y., Liang, X., Xiao, H., and Bi, K. (2003). Determination of spinosin in rat plasma by reversed-phase high-performance chromatography after oral administration of Suanzaoren decoction. J. Chromatogr. B. Analyt. Technol. Biomed. Life Sci. 787, 421-425. doi: 10.1016/S1570-0232(02)01001-2

Li, Y., Xu, B. Y., and Xiao, F. (2009). Effect of modified xiaoyao powder for improving sleep in patients with psychological stress insomnia. Chin. J. Integr. Tradit. West Med. 29, 208-211. doi: 10.3321/j.issn:1003-5370.2009. 03.006

Lian, F. M., Xu, G. C., Liu, K., Liu, Z. L., Wang, Q. F., Qiu, P., et al. (2009). Placebo controlled clinical trial of Chanyeanshen capsules in insomnia patients. Chin. J. New Drugs 18, 2056-2060. doi: 10.3321/j.issn:1003-3734.2009.21.014

Liu, L., Liu, C., Wang, Y., Wang, P., Li, Y., and Li, B. (2015). Herbal medicine for anxiety, depression and insomnia. Curr. Neuropharmacol. 13, 481-493. doi: 10.2174/1570159X1304150831122734

Liu, Y., and Nan, D. Y. (2009). Clinical observation on zao ren an shen capsule for psychophysiological insomnia. Chin. J. Chin. Mater. Med. 34, 1730-1731.

Long, J. (2010). Clinical study of ban-bai-zhen-hun Decoction on the Sleep Quality and Sleep Structure of Insomnia Patients, dissertation, Hunan University of Chinese Medicine, Hunan.

Lu, D. P. (2011). Clinical Study of Qing Xin zi shui Granule for Primary Chronic Insomnia Patients of Fire Hyperactivity Caused by Yin Deficiency. dissertation, Changchun University of Chinese Medicine, Changchun.

Ma, Y., Han, H., Eun, J. S., Kim, H. C., Hong, J. T., and Oh, K. W. (2007). Sanjoinine A isolated from Zizyphi Spinosi Semen augments pentobarbitalinduced sleeping behaviors through the modification of GABA-ergic systems. Biol. Pharm. Bull. 30, 1748-1753. doi: 10.1248/bpb.30.1748

Moher, D., Liberati, A., Tetzlaff, J., Altman, D. G., and PRISMA Group. (2010). Preferred reporting items for systematic reviews and meta-analyses: the PRISMA statement. Int. J. Surg. 8, 336-341. doi: 10.1016/j.ijsu.2010.02.007

Morin, C. M., Bootzin, R. R., Buysse, D. J., Edinger, J. D., Espie, C. A., and Lichstein, K. L. (2006a). Psychological and behavioral treatment of 
insomnia: update of the recent evidence (1998-2004). Sleep 29, 1398-1414. doi: 10.1093/sleep/29.11.1398

Morin, C. M., Leblanc, M., Daley, M., Gregoire, J. P., and Mérette, C. (2006b). Epidemiology of insomnia: prevalence, self-help treatments, consultations, and determinants of help-seeking behaviors. Sleep Med. 7, 123-130. doi: 10.1016/j.sleep.2005.08.008

Ni, X. J., Shergis, J. L., Guo, X. F., Zhang, A. L., Li, Y., Lu, C. J., et al. (2015). Updated clinical evidence of Chinese herbal medicine for insomnia: a systematic review and meta-analysis of randomized controlled trials. Sleep Med. 16, 1462-1481. doi: 10.1016/j.sleep.2015.08.012

Pan, W. H. (2011). Clinical Observation on Treatment of Zinianmeifang for Insomnia with Depressed Liver qi Transforming into Fire Syndrome. dissertation, Guangzhou University of Chinese Medicine, Guangzhou.

Peng, W. H., Hsieh, M. T., Lee, Y. S., Lin, Y. C., and Liao, J. (2000). Anxiolytic effect of seed of Ziziphus jujuba in mouse models of anxiety. J. Ethnopharmacol. 72, 435-441. doi: 10.1016/S0378-8741(00)00255-5

Ren, L., Wang, F., Xu, Z., Chan, W. M., Zhao, C., and Xue, H. (2010). GABA(A) receptor subtype selectivity underlying anxiolytic effect of 6hydroxyflavone. Biochem. Pharmacol. 79, 1337-1344. doi: 10.1016/j.bcp.2009. 12.024

Rodríguez, V. J., and Rodríguez, V. L. (2017). Experimental and Clinical Pharmacology of Ziziphus jujuba Mills. Phytother. Res. 31, 347-365. doi: $10.1002 /$ ptr.5759

Sarris, J., Panossian, A., Schweitzer, I., Stough, C., and Scholey, A. (2011). Herbal medicine for depression, anxiety and insomnia: a review of psychopharmacology and clinical evidence. Eur. Neuropsychopharmacol. 21, 841-860. doi: 10.1016/j.euroneuro.2011.04.002

Schunemann, H., Brozek, J., Guyatt, G., and Oxman, A. (2013). GRADE Handbook for Grading Quality of Evidence and Strength of Recommendations (The GRADE Working Group). Available online at: http://www.guidelinedevelopment.org/ handbook/

Shergis, J. L., Ni, X., Sarris, J., Zhang, A. L., Guo, X., Xue, C. C., et al. (2017). Ziziphus spinosa seeds for insomnia: a review of chemistry and psychopharmacology. Phytomedicine 34, 38-43.

Shi, M., Yang, D. D., and Zhao, H. (2014). Clinical study of Tang Yu Shu for insomnia with phlegm heat disturbing heart syndrome: 100 cases. Chin. Tradit. Pat. Med. 36, 1386-1389. doi: 10.3969/j.issn.1001-1528.2014.07.010

Singh, A., and Zhao, K. (2017). Treatment of insomnia with traditional chinese herbal medicine. Int. Rev. Neurobiol. 135, 97-115. doi: 10.1016/bs.irn.2017.02.006

Stegemann, S., and Bornem, C. (1999). Hard gelatin capsules today-and tomorrow. Capsugel. Libr. 1-23.

Trauer, J. M., Qian, M. Y., Doyle, J. S., Rajaratnam, S. M., and Cunnington, D. (2015). Cognitive behavioral therapy for chronic insomnia: a systematic review and meta-analysis. Ann. Intern. Med. 163, 191-204. doi: 10.7326/M14-2841

Wang, L. E., Cui, X. Y., Cui, S. Y., Cao, J. X., Zhang, J., Zhang, Y. H., et al. (2010). Potentiating effect of spinosin, a C-glycoside flavonoid of Semen Ziziphi spinosae, on pentobarbital-induced sleep may be related to postsynaptic 5-HT(1A) receptors. Phytomedicine 17, 404-409. doi: 10.1016/j.phymed.2010.01.014

Wang, S. M., Liu, J. Q., Ma, G. F., Lan, H. Y., and Zhao, Z. R. (2013). Clinical study of Zaorenanshen tablet for treating insomnia. Hebei J. Tradit. Chin. Med. 35, 1217-1219. doi: 10.3969/j.issn.1002-2619.2013.08.058

Wang, Z. T., He, M., and Jiang, J. (2010). Sanqi granule treatment of insomnia of deficiency heart blood and stagnant heat type: randomized doubleblind clinical curative effect observation. Liaoning J. Tradit. Chin. Med. 37, 2289-2292. doi: 10.13192/j.ljtcm.2010.12.22.wangzht.064
Wilt, T. J., Macdonald, R., Brasure, M., Olson, C. M., Carlyle, M., Fuchs, E., et al. (2016). Pharmacologic treatment of insomnia disorder: an evidence report for a clinical practice guideline by the American College of Physicians. Ann. Intern. Med. 165, 103-112. doi: 10.7326/M15-1781

Xie, C., Gu, Y., Wang, W. W., Lu, L., Fu, D., and Liu, A., et al. (2013). Efficacy and safety of Suanzaoren decoction for primary insomnia: a systematic review of randomized controlled trials. BMC Complement. Altern. Med. 13:18. doi: 10.1186/1472-6882-13-18

Yan, Z. H. (2010). Chinese Materia Medica, 2nd Edn. Beijing: People's Medical Publishing House.

Yang, B., Yang, H., Chen, F., Hua, Y., and Jiang, Y. (2013). Phytochemical analyses of Ziziphus jujuba Mill. var. spinosa seed by ultrahigh performance liquid chromatography-tandem mass spectrometry and gas chromatographymass spectrometry. Analyst 138, 6881-6888. doi: 10.1039/c3an $01478 \mathrm{a}$

Yeh, C. H., Arnold, C. K., Chen, Y. H., and Lai, J. N. (2011). Suanzaoren Decoction as an original treatment for sleep difficulty in climacteric women: a prospective clinical observation. Evid. Based Complement. Alternat. Med. 9 , 673-813. doi: 10.1155/2011/673813

Yeung, W. F., Chung, K. F., Poon, M. K., Ho, Y. Y., Zhang, S. P., Zhang, Z. J., et al. (2012). Chinese herbal medicine for insomnia: a systematic review of randomized controlled trials. Sleep Med. Rev. 16, 497-507. doi: 10.1016/j.smrv.2011.12.005

Yeung, W. F., Chung, K. F., Yung, K. P., Ho, F. Y., Ho, L. M., Yu, Y. M., et al. (2014). The use of conventional and complementary therapies for insomnia among Hong Kong Chinese: a telephone survey. Complement Ther. Med. 22, 894-902. doi: 10.1016/j.ctim.2014.08.001

You, Z. L., Xia, Q., Liang, F. R., Tang, Y. J., Xu, C. L., Huang, J., et al. (2010). Effects on the expression of GABAA receptor subunits by jujuboside A treatment in rat hippocampal neurons. J. Ethnopharmacol. 128, 419-423. doi: 10.1016/j.jep.2010.01.034

Yuan, C. X., Chen, Y., and Li, J. (2013). Adjuvant treatment of insomnia of liver-yin deficiency pattern by Mei'an grannule: a randomized double-blind and placobo-controlled trial. Shanghai J. Tradit. Chin. Med. 47, 57-59, 71. doi: 10.16305/j.1007-1334.2013.05.027

Zhang, M., Ning, G., Shou, C., Lu, Y., Hong, D., and Zheng, X. (2003). Inhibitory effect of jujuboside A on glutamate-mediated excitatory signal pathway in hippocampus. Planta. Med. 69, 692-695. doi: 10.1055/s-2003-42786

Zhao, K. (2013). Acupuncture for the treatment of insomnia. Int. Rev. Neurobiol. 111, 217-234. doi: 10.1089/acm.2009.0041

Zheng, X. Y. (1993). Guideline for Clinical Trials of New Patent Chinese Medicines, 1st Edn. Beijing: Ministry of Health of the People's Republic of China.

Zhou, J. L., Wang, H. G., Bu, L. L., and Dong, F. (2002). Double-blinded controlled study of self-made shen-an 3 capsule and estozolam for insomnia. Med. J. Chin. Civ. Adm. 14, 336-338. doi: 10.3969/j.issn.1672-0369.2002.06.005

Conflict of Interest Statement: The authors declare that the research was conducted in the absence of any commercial or financial relationships that could be construed as a potential conflict of interest.

Copyright (C) 2018 Zhou, Zhou, Xu, Jin, Rong, Zheng and Lin. This is an open-access article distributed under the terms of the Creative Commons Attribution License (CC $B Y)$. The use, distribution or reproduction in other forums is permitted, provided the original author(s) and the copyright owner are credited and that the original publication in this journal is cited, in accordance with accepted academic practice. No use, distribution or reproduction is permitted which does not comply with these terms. 